\title{
Valoración ecocardiográfica de la insuficiencia tricúspide secundaria
}

Paula Andrea Cuartas González

Unidad de cardiología no invasiva. Alianza cardiovascular. Hospital Universitario Mayor, MÉDERI. Bogotá DC. Colombia

\section{Correspondencia}

Paula Andrea Cuartas González pauliscuartis@gmail.com

\section{Recibido: 18/02/2018 \\ Aceptado: 14/01/2020 \\ En línea: 02/03/2020}

Citar como: Cuartas-González PA. Valoración ecocardiográfica de la insuficiencia tricúspide secundaria. Rev Ecocar Pract (RETIC). 2020 (Mar); 3 (1): 56-59. doi: 10.37615/retic.v3n1a15

Cite this as: Cuartas-González PA. Echocardiographic assessment of secondary tricuspid regurgitation. Rev Ecocar Pract (RETIC). 2020 (Mar); 3 (1): 56-59. do: 10.37615/retic.v3n1a15

\section{Palabras clave}

$\triangleright$ Técnico en ecocardiografía

$\triangleright$ Hallazgos ecocardiográficos

$\triangleright$ Insuficiencia tricúspide secundaria

Keywords

$\triangleright$ Cardiac sonographer

$\triangleright$ Echocardiography findings

$\triangleright$ Secondary tricuspid regurgitation

\section{RESUMEN}

La valoración ecocardiográfica es la piedra angular en el diagnóstico de la insuficiencia tricúspide secundaria, de hecho el ventrículo derecho y la válvula tricúspide son estructuras que a lo largo del tiempo han ganado interés en su estudio, como posibles causas de insuficiencia cardíaca. La reciente incorporación de la posibilidad de corrección percutánea de la regurgitación tricúspide ha hecho que la valoración de este tipo de patología haya cobrado una mayor relevancia en la práctica clínica. Esta patología valvular requiere un abordaje multidisciplinar realizado por un Heart Team, donde el técnico en ecocardiografía y el cardiólogo especialista en imagen tienen un papel determinante en el diagnóstico y seguimiento

ABSTRACT
Echocardiographic assessment is the cornerstone, in the diagnosis of secondary tricuspid regurgitation the-
refore, the right ventricle and the tricuspid valve are structures that over time have gained interest in their
study as possible causes of heart failure. The recent incorporation of the possibility of percutaneous correc-
tion of tricuspid regurgitation, implies that the assessment of this type of regurgitation is more important
in clinical practice. This valvular pathology requires a multidisciplinary approach performed by a Heart
Team where the cardiac sonographer and cardiac imaging specialist have an important role in diagnosis
and follow-up.

Anatomía de la válvula tricúspide

La válvula tricúspide es la válvula auriculoventricular con mayor área y tiene una implantación más apical que la válvula mitral. Está soportada sobre una compleja estructura anatómica denominada complejo valvular tricúspide, compuesto por anillo tricúspide, valvas (anterior, posterior y septal), cuerdas tendinosas, músculos papilares (anterior, posterior y septal), pared libre del ventrículo derecho y banda moderadora.

Esta válvula se caracteriza por la presencia de múltiples sitios de fijación, sus cuerdas tendinosas pueden fijarse a dos músculos papilares simultáneamente, a la pared ventricular y a la banda moderadora.

El anillo tricúspide es más grande que el anillo mitral, tiene un papel fundamental en la arquitectura valvular, da firmeza a la válvula en los puntos de inserción de las valvas y tiene estructura elíptica en silla de montar.
Etiología de la insuficiencia tricúspide secundaria

La etiología secundaria o funcional es frecuente (un $75 \%$ de los casos). La insuficiencia tricúspide puede ser secundaria a la presencia patología valvular izquierda, distintas miocardiopatías y enfermedades pulmonares: se produce dilatación del anillo tricúspide, contracción asimétrica, dilatación de cavidades derechas e hipertensión pulmonar

\section{Valoración ecocardiográfica de la insuficiencia tricúspide secundaria o funcional}

La valoración ecocardiográfica de la válvula tricúspide exige el uso de múltiples planos para hacer una valoración global de su anatomía. La ecocardiografía transtorácica es el método de elección para el abordaje inicial de 
esta valvulopatía y determina el uso de técnicas complementarias de imagen cardíaca.

Es importante que los técnicos de ecocardiografía conozcan los mejores tru cos para obtener todos los hallazgos ecocardiográficos presentes en la insuficiencia tricúspide secundaria. A continuación se describen algunos pasos que permitirán obtener una valoración ecocardiográfica secuencial y sistemática de esta patología valvular.

\section{Paso 1. Valoración anatómica de la válvula tricúspide}

En este paso se identifican las valvas comprometidas, la presencia de prolapso, flail, restricción, tenting, el grosor de las valvas, la presencia de calcio, vegetaciones y se analiza el mecanismo de coaptación. Además, se identifica la presencia o no de electrodo de estimulación de dispositivos, su ubicación y posible afectación del mecanismo de coaptación valvular.

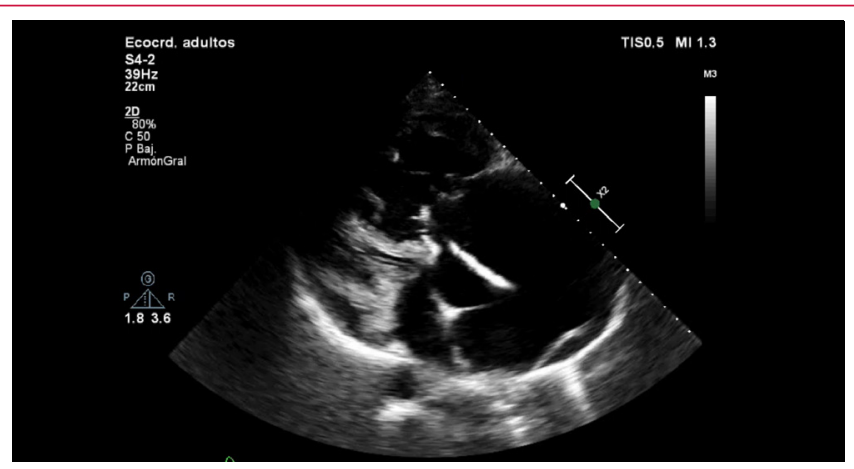

Vídeo 1. Visualización de electrodo de dispositivo desde tracto de entrada del ventrículo derecho

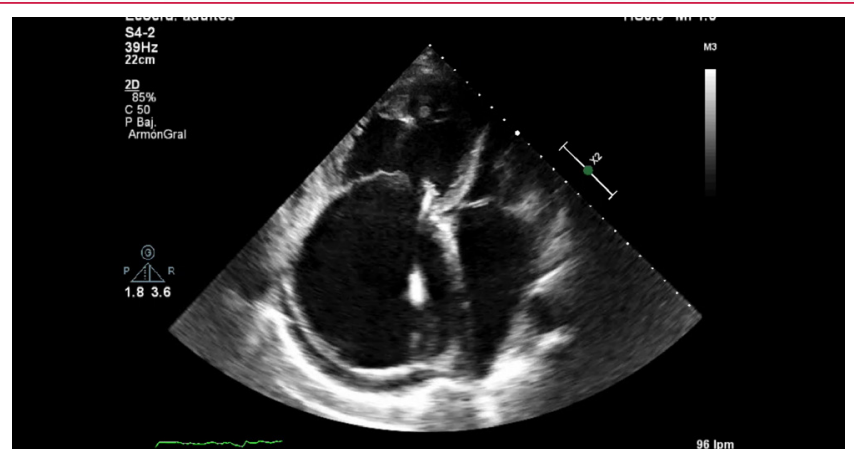

Vídeo 2. Visualización de electrodo de dispositivo. Plano apical de cuatro cámaras, énfasis en ventrículo derecho

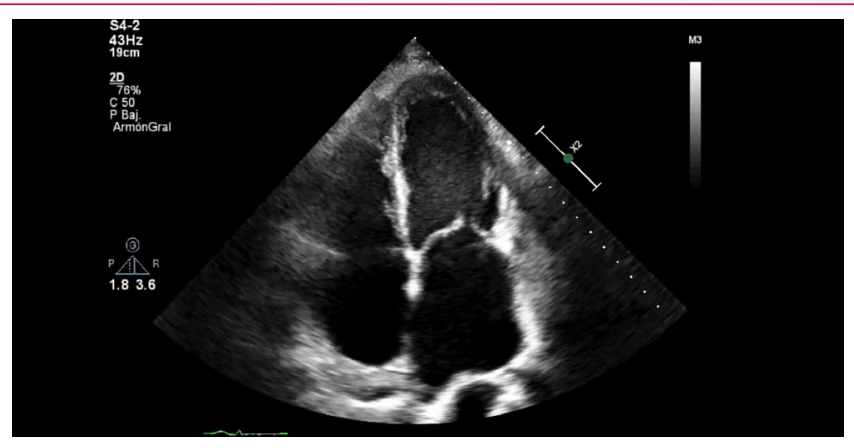

Vídeo 3. Valoración de amplio defecto de coaptación tricuspidea. Plano apical de cuatro cámaras
Paso 2. Valoración de las características del jet de regurgitación

En este paso hay que identificar la dirección del jet de regurgitación y su relación con estructuras anatómicas adyacentes como el septum interauricular, el techo y las paredes de la aurícula derecha. Además, hay que valorar si se observa la presencia de efecto coanda y el número de jets de regurgitación que se observan, según las diferentes proyecciones ecocardiográficas obtenidas.

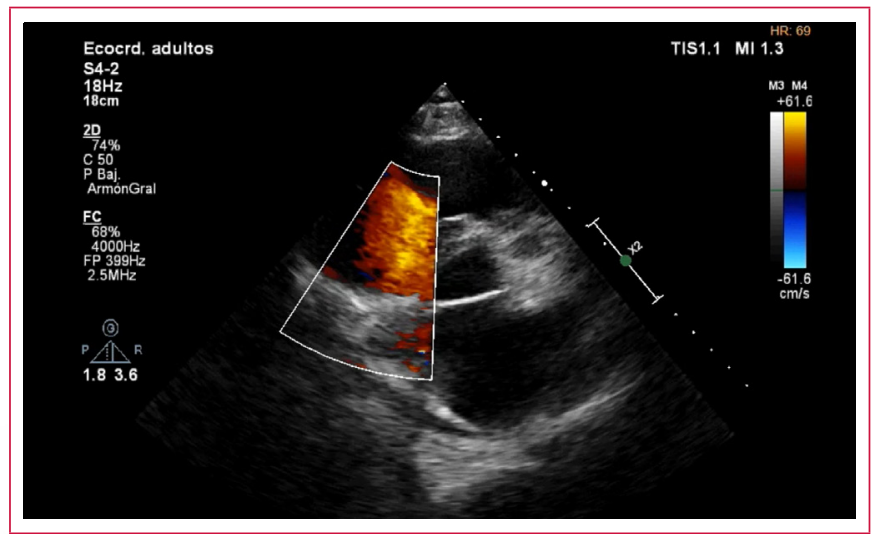

Vídeo 4. Doppler color: insuficiencia tricúspide desde el eje corto a nivel de grandes vasos modificado, visualización de las tres valvas tricúspides

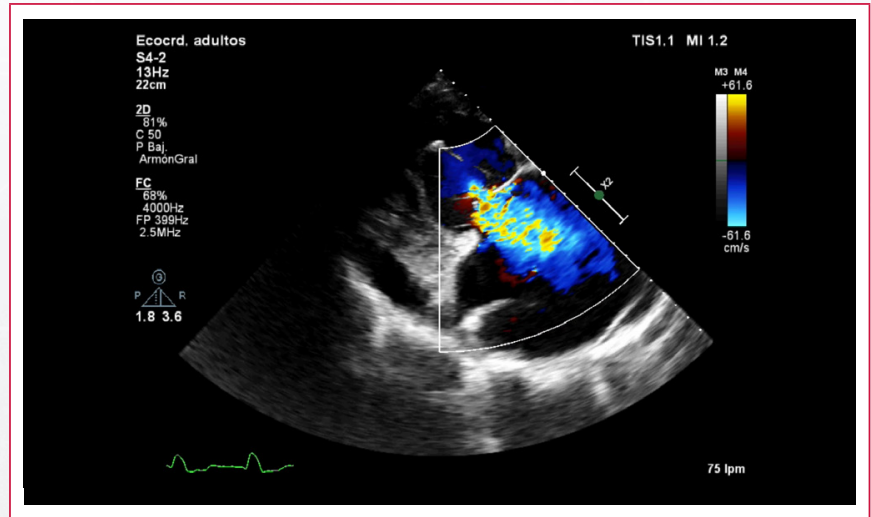

Vídeo 5. Doppler color. Insuficiencia tricúspide desde el tracto de entrada del ventrículo derecho

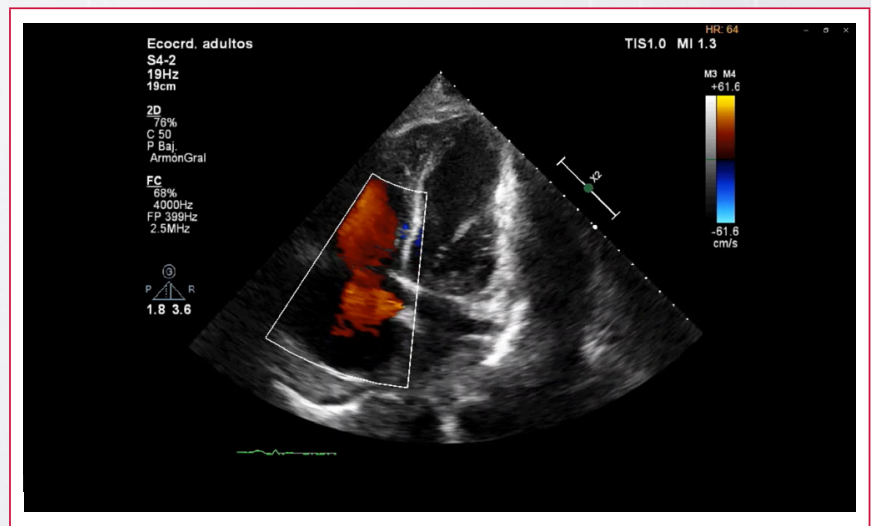

Vídeo 6. Doppler color. Insuficiencia tricúspide desde el plano apical cuatro cámaras, énfasis en ventrículo derecho 


\section{Paso 3. Valoración del tamaño del anillo tricúspide}

La medición del anillo se hace en una imagen de zoom bidimensional con uso de sincronización electrocardiográfica, en el plano apical de cuatro cámaras en la inserción de las valvas. Se busca la telediástole y se traza una línea de extremo a extremo lo más perpendicular posible a la estructura, teniendo precaución de no incluir la pared ventricular. Un diámetro del anillo en diástole mayor a $34 \mathrm{~mm}$ es un marcador de insuficiencia tricúspide significativa. Existe una correlación entre el tamaño del anillo tricúspide y la severidad de la insuficiencia, por tal motivo es muy importante su adecuada medición de forma rutinaria.

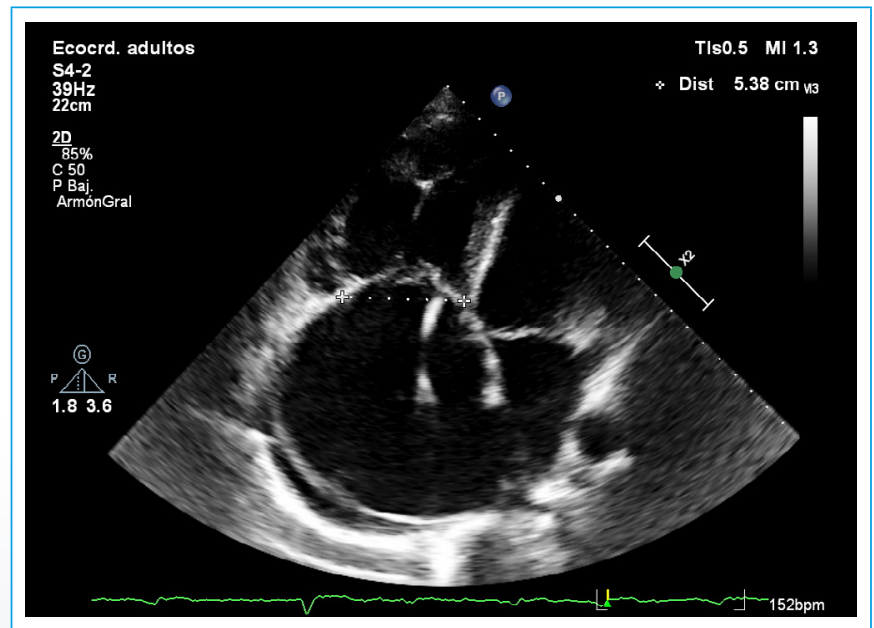

Figura 1. Medición del anillo tricúspide

Si se identifica dilatación del anillo, también se puede medir el área de tenting, este parámetro ayuda a identificar el grado desplazamiento apical de las valvas secundario a la dilatación del anillo y del ventrículo derecho. Un área de tenting mayor a $1 \mathrm{~cm}^{2}$ está asociado con insuficiencia tricúspide significativa.

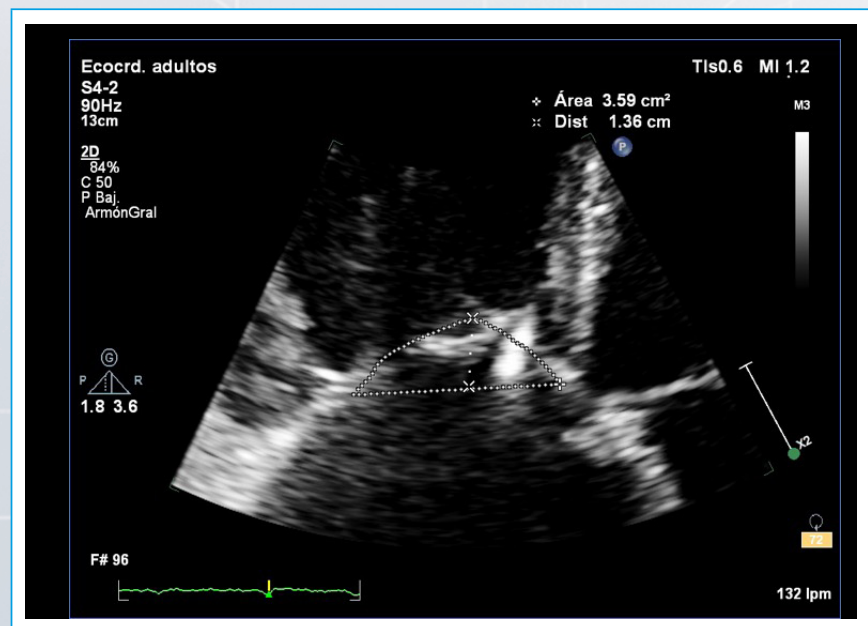

Figura 2. Medición del área de tenting de la válvula tricúspide

\section{Cuantificación de la severidad de la insuficiencia}

En la actualidad se han descrito el uso de múltiples parámetros para estimar la severidad de las insuficiencias. Es importante estar familiarizado con éstos y saber cómo adquirir las mejores imágenes para su adecuada estimación. A continuación se mencionan los parámetros más frecuentemente utilizados:
- Área del jet de regurgitación.

- Radio de PISA y ORE.

- Volumen regurgitante y fracción regurgitante.

\section{Recomendaciones técnicas para el adecuado registro de parámetros ecocardiográficos durante la valoración de la insuficiencia tricúspide secundaria}

Recomendaciones técnicas para la medición del área del jet de regurgitación

1. Optimización del límite de Nyquist del equipo (entre 50 y $70 \mathrm{~cm} / \mathrm{s}$ ).

2. Optimización de la ganancia del color (evitar señales con ruido que tienden a sobreestimar la magnitud del área del contorno del jet).

3. Esta medición se afecta por la atenuación de la señal de ultrasonido por mala ventana acústica y por la presencia de reflectores.

4. Preferir transductores con bajas frecuencias.

5. Tener precaución con las señales del jet de regurgitación muy intensas o muy débiles, lo que puede crear confusión en el momento de la medición.

6. Obtener una alineación del jet lo más paralelo al flujo posible. Para esto en muchas ocasiones es necesario realizar movimiento de angulación del transductor y el uso de proyecciones no convencionales.

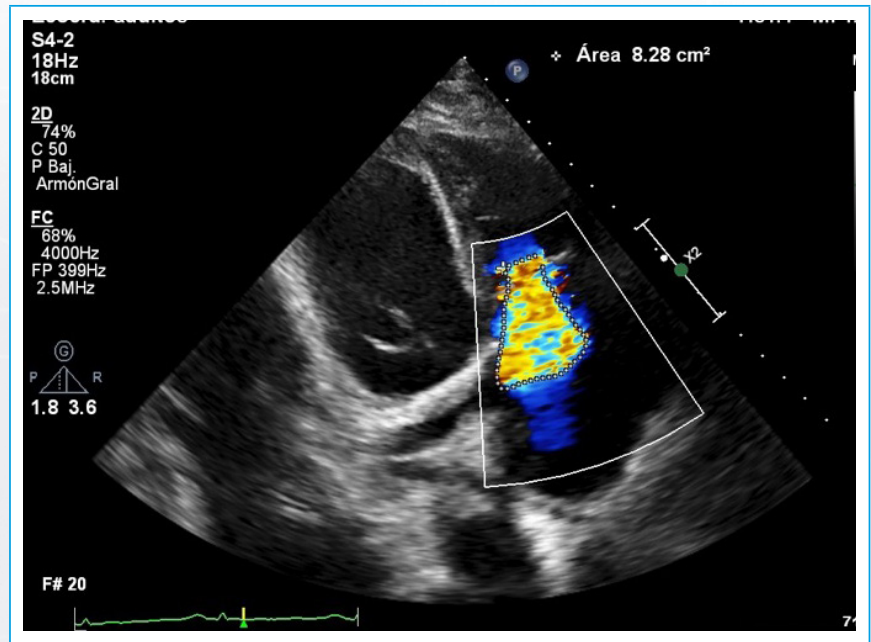

Figura 3. Medición del área del jet de regurgitación

Recomendaciones técnicas para la obtención de imágenes para medición del radio PISA y cálculo del ORE

1. Obtener una imagen de zoom bidimensional color sincronización electrocardiográfica, en la proyección de mejor alineación del jet de regurgitación, bajar la línea de base del Doppler color entre 30 a $50 \mathrm{~cm} / \mathrm{s}$ del límite de Nyquist y adquirir la imagen de al menos tres latidos consecutivos.

2. Optimizar el tamaño de la ventana del color en donde sólo se incluya los tres componentes del jet de regurgitación: hemiesfera, vena contracta y extensión del jet.

3. Identificar la zona de isovelocidad con los tres componentes del jet de regurgitación, identifica la hemiesfera en la primera línea de aliasing teniendo en cuenta el cambio del mapa de color, de colores rojos a amarillos en mitad de sístole. 
4. Medir el radio de PISA partiendo del punto más estrecho del jet de regurgitación que generalmente concuerda con el punto de coaptación valvular, extender la medición hasta el primer contorno del aliasing.

5. Una vez obtenido el radio de PISA, integrar al paquete de cálculos la velocidad de aliasing y el IVT del jet de rergurgitación para la estimación del ORE.

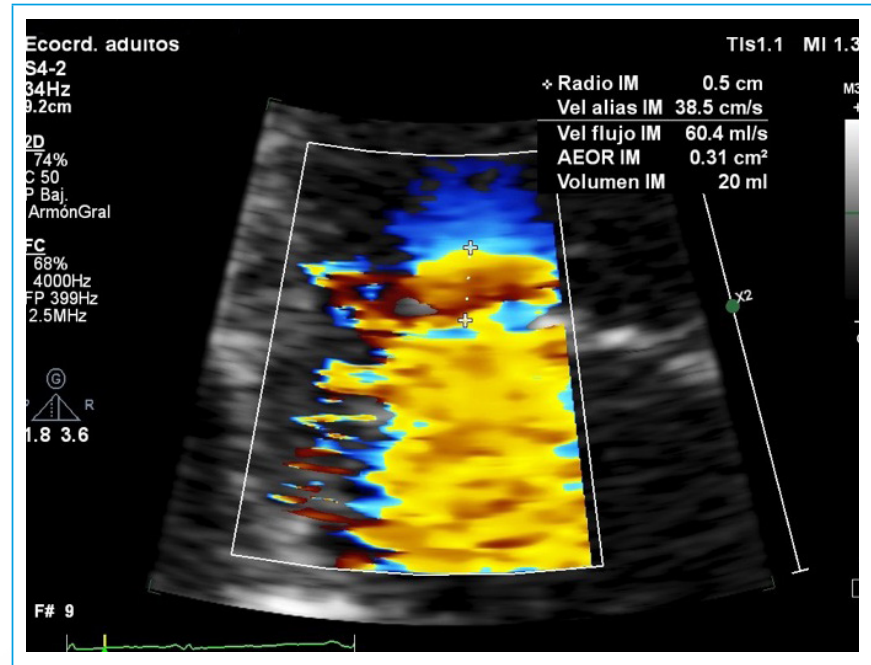

Figura 4. Medición del radio de PISA

Recomendaciones técnicas para la obtención de registros espectrales para cálculos hemodinámicos mediante Doppler continuo

1. Para la obtención la ITV es necesario una correcta alineación con el flujo garantizar que ésta sea lo más paralela posible al mismo.

2. Ajustar los filtros de rechazo del Doppler pulsado que deben estar al mínimo.

3. Ajustar la ganancia de la imagen espectral para evitar sobre estimación del parámetro.

4. El trazado de la ITV exige extrema precisión, no salirse del contorno de la envoltura, y evitar trazos irregulares.

5. Es necesario optimizar la escala de la imagen espectral que permita obtener una ITV fácil de trazar que evite errores, si la imagen espectral es muy pequeña o la velocidad de barrido no es la adecuada, se pueden magnificar los errores durante el trazado.

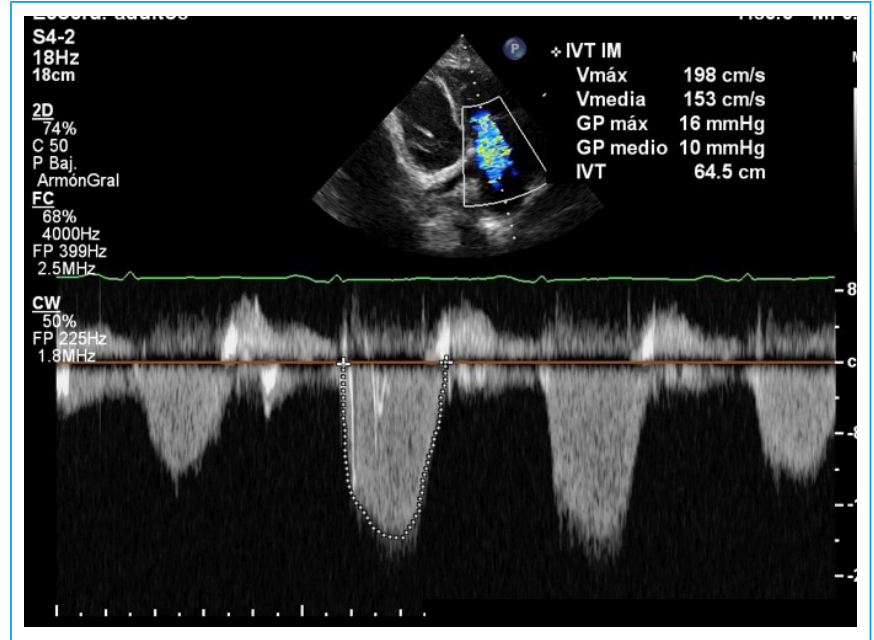

Figura 5. Imagen espectral jet de insuficiencia tricúspide

\section{Ideas para recordar}

- La válvula tricúspide tiene características anatómicas y fisiológicas que es necesario conocer para su adecuada valoración.

- La insuficiencia tricúspide secundaria o funcional es una patología compleja de alta incidencia.

- La valoración con ecocardiografía transtorácica es una piedra angular en el diagnóstico.

\section{Bibliografía}

1. Badano LP, Muraru D, Enriquez-Sarano M. Assessment of functional tricuspid regurgitation. Eur Heart J 2013; 34: 1875-1885.

2. Lancellotti P, Moura L, Pierard LA, et al. European Association of Echocardiography recommendations for the assessment of valvular regurgitation. Part 2: mitral and tricuspid regurgitation (native valve disease). Eur J Echocardiogr 2010; 11: 307-332.

3. Zoghbi WA, Adams D, Bonow RO, et al. Recommendations for Non-invasive Evaluation of Native Valvular Regurgitation: A Report from the American Society of Echocardiography Developed in Collaboration with the Society for Cardiovascular Magnetic Resonance. J Am Soc Echocardiogr 2017; 30: 303-371. 